\title{
Effects of Discourse Markers on EFL Reading: A Case Study
}

\author{
Shao-Yang Luo \\ English Department, School of Foreign Languages \\ Jimei University \\ Xiamen, China
}

\author{
Jiao Dong \\ English Department, School of Foreign Languages \\ Jimei University \\ Xiamen, China
}

\author{
Dong-Hong Han \\ English Department, School of Foreign Languages \\ Jimei University \\ * Corresponding author
}

\begin{abstract}
Despite many studies on discourse markers (DMs), how DMs affect EFL learners' reading has been neglected as a research topic. This paper carries out an investigation into the influence of discourse markers on reading among the first-year non-English majors from the perspective of Relevance Theory, with an attempt to improve English teaching and students' EFL reading ability. A reading test among $\mathbf{1 3 5}$ college freshmen is conducted and their scores are collected and analyzed by SPSS. Findings show that there is no significant difference between students' reading performance from their answers with the guidance of DMs and without the guidance of DMs. The result indicates that discourse markers have little influence on these first-year non-English majors' reading, which can be seen as a problem existing in English teaching. Based on these findings, suggestions are offered to improve EFL learners' reading comprehension.
\end{abstract}

Keywords-discourse markers; Relevance Theory; influence; English reading

\section{INTRODUCTION}

Discourse markers refer to those words that signal the logical relationships and contribute to the coherence of discourse in a text. With the development of pragmatics, studies on discourse markers (DMs) have gradually come into people's view since the 1970s. However, most studies focus on the definitions, classification, and functions of discourse markers[1]. Some researchers[2-3] study on individual DMs while others treat all DMs as a whole [4-6]. Nonetheless, no consensus has been reached as to the definition or the classification of discourse markers. Despite the complexity of DMs study, fruitful results have been yielded due to its wide range and multiple functions. Ran [7] pointed out that the study of DMs, since the 1970s, has been turning from the perspective of syntactic/semantic--pragmatic" to the perspective of "pragmatic-cognitive". In other words, not only has the study of DMs facilitated the development of syntax

The present study is financially supported by Foundation for Innovative Research in Teaching Undergraduate Students (JY18106) and Postgraduate Students (YJG0817) of Jimei University and Foundation for Academic Research Project (HW18138) supported by Foreign Language Teaching and Research Press. and semantics, but it also has promoted the study of pragmatics and cognitive linguistics. In China, many researchers [8-9] have also studied DMs in English and much attention has been paid to studies of DMs used by EFL learners in writing[10-11] and listening [12-13], while few reports have been revealed on those of EFL reading. Some studies indicate that linguistic markers improve the mental text representation, for example, with better answers on comprehension questions[14], as readers can benefit from the presence of causal relational markers both in L1 and in L2 [15]. However, other studies reveal that linguistic marking of coherence relations does not bring better answers to comprehension questions [16]. Some researchers even claim a negative impact of connectives on text comprehension [17]. Now that no consensus has been reached as to the effects of discourse markers on reading comprehension, it is necessary to carry out more studies on this topic. Therefore, within the framework of Relevance Theory, the present study carries out an investigation into effects of discourse markers on EFL reading of the first-year non-English majors, with an attempt to contribute to the development of DMs study, shed light on current EFL pedagogies, and provide constructive implications for EFL learners and teachers.

\section{THEORETICAL FRAMEWORK}

\section{A. Relevance Theory}

Relevance, according to Sperber and Wilson[18], is the key to human cognition. Their account of communication results from the belief that humans tend to pay attention to those phenomena that are most relevant to them. Relevance is treated as a property of inputs to the cognitive process and analyzed in terms of the notion of cognitive effect and processing effort. On the other hand, when an item of input (for example, an utterance) is processed in a context of available assumptions, it may yield some cognitive effects (or contextual effect). An utterance can have contextual effect in one of the three ways: New information may provide further evidence for, and therefore strengthen old assumptions; new information may provide evidence against, and perhaps lead to the abandonment of old assumptions; new and old information 
may interact with each other, and therefore produce contextual implications.

Relevance also has degrees determined by the effect and effort that the speaker and hearer pay. The contextual effects of an assumption in a given context are not the only factor to be taken into account in assessing its degree of relevance. Contextual effects are brought about by mental process which involves a certain effort, the processing effort in interpreting utterance. Processing effort is a negative factor: other things being equal, the greater the processing effort, the lower the relevance.

Relevance Theory accounts for communication as dynamic interaction between the speaker and the hearer, so it provides a general explanation of what discourse markers employed in communication are functioning cognitively and pragmatically.

\section{B. DMs and Relevance Theory}

According to Relevance Theory, human communication is relevance-oriented. A speaker or writer, by making an utterance, makes his/her claim that it will be worthwhile to process this utterance. This provides the hearer or reader with a guarantee that the speaker has aimed to produce an utterance that yields adequate contextual effects, and thus entitles the hearer to interpret the utterance in the smallest context at the minimum cost. This means that if the speaker wishes the hearer to recover his/her intended interpretation, he/she has to constrain the hearer's interpretation and direct the hearer to a particular set of assumptions, and the hearer should try to recognize the speaker's intended interpretation. The fewer contextual assumptions the hearer can select, the more relevant an utterance will be; and the lower the processing effort needed, the greater the relevance. Hence, relevance is determined by the relation between contextual effects gained and the processing effort required. This hypothesis can be applied to DMs. In the relevance-based approach [19], DMs are seen as semantic constraints on relevance. They do not encode conceptual meaning, but play a procedural role in the referential process of comprehension, constraining the range of possible interpretations of the utterance and hence reducing the processing effort required. Due to the speaker's constraints on the hearer's choice of contextual assumptions by using DMs, the hearer interprets utterances in the smallest and most accessible context that yields adequate contextual effects for no unnecessary efforts. Therefore, Relevance Theory provides a strong framework to explain the motivation for the use of DMs in communication and interpretation that cannot be explained by other pragmatic theories in this domain.

\section{RESEARCH DESIGN}

\section{A. Research Purposes}

The present study is to investigate whether discourse markers contribute to EFL learners' overall understanding of a text, and if so, to what extent, if not, why. In addition, the study is to find out whether the learning of discourse markers is one of the key elements in the improvement of students' language development in EFL settings.

\section{B. Research Questions}

In this study, we shall find answers to the following questions.

- Do DMs help readers interpret the text?

- What is the difference between the accuracy of their answers to questions designed with and without the guidance of DMs?

- How do DMs have effects on the first-year non-English majors' reading comprehension?

\section{Participants}

To investigate how DMs affect readers in text understanding, 135 first-year non-English majors were chosen to take a reading test in an English class. We chose these non-English majors at random as the objects of this study for three reasons. First, they are different majors who can represent the English level of freshmen. Second, it is their first year in university and they have little impression of higher English learning skills. Finally, it is good for them to be the objects because collecting the research data from them makes it possible to find out and solve their problems in EFL reading from the very beginning as college students.

\section{Procedures}

In this case study, we conducted an experiment of EFL reading tests to find out the effect of DMs on reading. Our data are collected in three steps. First, the reading test consists of 2 passages from College English of Band I, with 5 multiple questions for each. Questions for both the passages consist of those which are related to DMs. Among these 10 questions, one half of them are answered with the guidance of discourse markers, and the other half are not. Then, the test was conducted among 135 first-year non-English majors in a College English class. The students were asked to finish their reading and answer 10 questions within 20 minutes. The return rate was $100 \%$, however, 11 students didn't finish their test, so only 124 students' answers were valid and used as the data of this study. Finally, the accuracy of each question is collected and recorded in tables (see Table 1 and Table 2), and the data are statistically analyzed by SPSS 25.0 with Independent Samples T-Test.

\section{RESULT AND DISCUSSION}

\section{A. Result}

TABLE I. DISCOURSE MARKERS FOR EACH QUESTION IN THE READING TEST

\begin{tabular}{|c|c|c|}
\hline Passages & Question No. & DMs \\
\hline \multirow{4}{*}{ Passage 1 } & 1 & none \\
\cline { 2 - 3 } & 2 & none \\
\cline { 2 - 3 } & 3 & but \\
\cline { 2 - 3 } & 4 & On the other hand \\
\cline { 2 - 3 } & 5 & By no means \\
\cline { 2 - 3 } & 6 & none \\
\cline { 2 - 3 } & 7 & In particular \\
\cline { 2 - 3 } & 8 & none \\
\cline { 2 - 3 } & 9 & none \\
\hline
\end{tabular}


Table I shows all the discourse markers for each question in the reading test. It can be seen that in the reading test for the first-year non-English majors used in the present study, there are altogether 10 questions for 2 passages, among which the answers to 5 questions are based on the understanding of DMs, while the others are not. To be specific, the answers to Question No. 3, 4, 5, 7, and 10 are not related to DMs, while the answers to Question No. 3, 4, 5, 7, and 10 are based on the guidance of discourse markers but, on the other hand, by no means, in particular, and actually, respectively. These markers are assumed to help the students understand the passages and answer the questions correctly.

TABLE II. ACCURACY OF FIRST-YEAR NON-ENGLISH MAJORS ANSWERS IN READING

\begin{tabular}{|c|c|c|c|}
\hline Questions & Question No. & Accuracy (\%) & $\begin{array}{c}\text { Average } \\
(\%)\end{array}$ \\
\hline \multirow{5}{*}{$\begin{array}{l}\text { Without } \\
\text { markers }\end{array}$} & 1 & 40 & \multirow{5}{*}{60} \\
\hline & 2 & 86 & \\
\hline & 6 & 21 & \\
\hline & 8 & 62 & \\
\hline & 9 & 91 & \\
\hline \multirow{5}{*}{$\begin{array}{l}\text { With } \\
\text { markers }\end{array}$} & 3 & 68 & \multirow{5}{*}{55.6} \\
\hline & 4 & 36 & \\
\hline & 5 & 40 & \\
\hline & 7 & 70 & \\
\hline & 10 & 64 & \\
\hline
\end{tabular}

Table II shows the accuracy of 124 students' answers to each question in the reading test. It can be seen that the highest accuracy (above 80\%) occurs to question No. 9 and No.2 (non-marker questions), with $91 \%$ and $86 \%$, respectively. In contrast, two answers with the clue of DMs - No. 4 and No.5 are found with the lowest accuracy, $36 \%$ and $40 \%$, respectively Question No.7 and No.10 which can be answered with the guidance of DMs have an accuracy of only $70 \%$ and $64 \%$, respectively. The performance of two types of questions is both at comparatively low level, with an average of $60 \%$ and $55.6 \%$, respectively.

The result of Independent Samples T-Test of students reading accuracy by SPSS 25.0 shows that there is no significant difference $(p=0.782>0.05)$ between the accuracy of these 2 types of questions, indicating that DMs have hardly any effects on these first-year non-English majors' EFL reading. No matter whether they are guided with DMs or not, these first-year non-English majors' reading accuracy is at comparatively low level.

\section{B. Discussion}

The result of the average accuracy of the reading test $(66 \%$ and 55.6\%) indicates that first-year non-English majors' EFL reading is at a comparatively low level. An explanation for this result is that the test was taken at the beginning of the first term of these non-English majors. Due to long summer vacation, without reading or practicing English, many students forgot a lot in vocabulary. In addition, non-English majors' levels are comparatively low in general, esp. in reading, which is reported in many previous studies [20]. The third reason may lie in the limited number of questions used in this test.

The result also shows that there is no significant difference in the accuracy of answering questions with the clue of DMs and questions without the clue of DMs. This result seems to be unexpected and contrast to our assumption. As we have discussed above, DMs can facilitate the hearer's processing by indicating the direction in which relevance is to be sought in virtue of referential connections they express [19]. According to Relevance Theory, DMs can help readers understand the author's intention better. There are some reasons that may account for this unexpected result. Firstly, the participants took the test at the beginning of their first term as college students, and it is possible that before they went to college they received little training as to how to use DMs to help their understanding in the reading comprehension, so they need more knowledge and need to increase their awareness about DMs. The second possible reason lies in our limited samples, for the reading test used in the present study consists of only two passages, with only five questions for each. Another possible explanation is that the test was conducted right after summer vacation, during which most students hardly spent time in learning English, thus making their accuracy comparatively lower. The last possible reason for this result may be related to the questions designed for reading. Among these reasons, the most important one is that while reading, many learners didn't know what to do with DMs, let alone get the clue that the DMs provided for them. The following examples are given to illustrate this point:

(1) What does Paul Saffo mean in Paragraph 4 when he says, "...all you can do is press your nose to the window and watch?" a) TV is the greatest symbol of the mass media

b) Watching TV at home, you can know about the world

c) People can't get addicted to TV

d) TV turns people into passive watchers

(Passage 1 question 4)

The answer can be found among this part of Passage 1:

He suggests that the greatest symbol of mass media was the $T V$. "It delivers the world to your living room, but all you can do is press your nose to the window and watch," he says. New technology, on the other hand, makes each person more active. Recording television programs on a computer allow users to edit out commercials and watch the programs anytime they choose. With music, people can access one song at a time and build their own albums.

This question can be very easy if readers notice the discourse marker on the other hand from the coming discourse. Obviously here it implements a high-level of speech act - a contrast. From the perspective of Relevance Theory, the marker functions as a signpost for the readers thus reducing the processing effort in interpreting the writer's intentional meaning. Therefore, from the sentence "New technology, on the other hand, makes each person more active", we know the above paragraph is talking about a negative way about new technology-making us passive. So for this question, we should choose d) - TV turns people into passive watchers. If the students know the function of on the other hand, most of them won't choose the wrong answer. Here the marker gives a sign to the readers in interpreting the contextual implication, so the processing effort can be reduced to the least to understand the author's intention. With the guidance of this marker, it is easy for readers to choose the correct answer. However, $66 \%$ of the 
students ignored it, or misread the passage and got the wrong answer.

(2) What does Paul Saffo feel about the future of technology (Passage 1 question 5)

a) Worried

b) Hopeful

c) Confident

d) Excited

The answer can be found among this part of Passage 1:

He (Paul Saffo) is by no means certain that this is entirely a good thing. "The new technology is so bewitching that it risks turning everything into entertainment ... and the lesson from Rome onwards is that all great civilizations fail by turning everything into entertainment."

The discourse marker by no means is an emphasized word used to demonstrate the writer's strong emotion, meaning absolutely not. From this we know Paul Saffo is not positive about this technology, so the answer will be a negative word worried. As long as one understands this marker, he/she can make the correct choice. However, it is a pity that $60 \%$ students failed in understanding this discourse marker by no means or just neglected it, thus giving the wrong answer.

(3) According to the passage, what plays a particular role in bringing about the Web culture? (Passage2 question7)

a) Young people

b) The United States

c) Online business

d) College education

The answer lies in the sentence of Passage 2:

"Colleges, in particular, are providing the basis for a Web-ready culture." The marker in particular means particularly, and it is also a strong word to demonstrate the author's strong feeling about the college's particular role in bringing about the Web culture, which clearly indicates the author's true attitude. Therefore, it is not surprising the accuracy of the performance of this question was as high as $70 \%$, though still there are $30 \%$ students who failed to give the correct answer.

(4) How will people find time for the net? (Passage 2 question10)

a) The net will actually save them time

b) The net will provide excellent traffic flow

c) The net will offer shares of time to buy

d) The net will become quicker than in the past

The answer can be found among this part of Passage 2:

"In some instances, people will actually save time because the Web will make doing things better and more quickly than in the past."

Here the discourse marker actually indicates an opposite opinion from previous information. It emphasizes a different opinion from the ordinary people - Web will waste our time. Therefore, the author means saving time instead of wasting time. This is also supported by Ran [7], who states that discourse markers signal to the hearer... whether utterances follow smoothly from what has been uttered before, or whether some kind of disjunction is occurring. Obviously, in this example, the author employs actually to show the reader that a disjunction is followed in the coming discourse. Yet there are still $36 \%$ of the students who gave a wrong choice.

It can be seen from the above examples that the impact of DMs in reading shouldn't be ignored, however, most of our students are lack of knowledge on DMs, or they don't know how to use them as guidance in reading comprehension. Considering the roles discourse markers play in helping readers interpreting the meaning and the limited knowledge our students have about DMs, it is of great significance to train the students with discourse markers in EFL learning.

\section{Pedagogical Implications}

Discourse markers show the relationship between the forgoing and upcoming discourse, and they "pragmatically function as guidelines or as road signals to suggest to the hearer a preferred line of interpretation of the ongoing utterances in conversation" [7]. In reading, these markers reveal the relationship the writer perceives between what he/she writes and the relative importance he/she attaches to it and other things about his/her attitude. It is often the case that when the reader understands one part, it serves as a key to the other part. Though one may not fully realize what the writer's intention is, at least to some degree, it is revealed in what he/she writes. Discourse markers of this kind indicate the functional value of sentences in which they occur. It thus has useful implications for teaching EFL reading:

1) Help EFL learners infer the meaning of unknown words. EFL learners of an intermediate level frequently meet difficult or unknown words when they are engaged in reading tasks. Some of them may even get frustrated because these difficult words are often a hindrance for correct text comprehension. It is necessary for teachers to teach students how discourse markers work in order to help EFL learners understand the functions that discourse markers play in a text, thus facilitating their reading and improving their reading comprehension.

2) Help EFL learners to locate the writer's view while reading. Writers always have certain audiences in mind when they write. Their works generally have very clear purposes and target readers. But this is not the case with the reader: as readers, we have different purposes in reading. It sometimes leads to a different understanding of the same text, which is not rare in the classroom. Teachers' task is to help students see what the writer's intended meaning is and eliminate other possible interpretations.

As was illuminated above, discourse markers are often employed by writers to show their intentions. If a writer thinks there should be a contrast, however or on the contrary may be used; if there are some statements to be clarified, in a nutshell, or that is to say may be employed. The writer may not be doing all these intentionally, but in any case, he/she can't avoid using them. To interpret a text as the writer intends doesn't mean there is only one meaning, but that good reader can always interpret a text as the writer intends it to be. Therefore, it is advisable for teachers to attach great importance to discourse markers in EFL class. As EFL learners, it is essential for them 
to learn more about discourse markers, practice more in speaking and writing so as to increase their awareness of DMs while reading, and finally improve their communicative competence.

\section{CONCLUSION}

Based on Relevance Theory, this paper studies the effects of discourse markers on EFL reading comprehension through a reading test among first-year non-English majors in a university of China. The accuracy of the test reveals that first-year non-English majors are mostly at comparative low level (60\% and $55.6 \%$ in accuracy) in EFL reading, which indicates that great efforts should be made to improve their reading ability. The analysis of DMs within the perspective of Relevance Theory indicates that discourse markers do help readers understand text meaning, however, Independent Samples T-Test by SPSS 25.0 reveals that there is no significant difference between these non-English majors' reading with the guidance of DMs and without the guidance of DMs, which indicates discourse markers do not have any great influence on their text interpretation.

The possible reasons for these results are found in five aspects. First, the test is taken at the beginning of the first term of non-English majors, when they have limited knowledge about DMs. Second, the limited number of participants, as well as questions, is another possible explanation for the low accuracy. Third, as first-year non-English majors, it is possible that they don't know or they don't have the awareness about how to use DMs in reading. Another possible reason is that the questions in the reading test of this study are limited in number. The last possible explanation is that the questions in the test may have not been perfectly designed for this study. The findings imply that more attention should be given to reading practice, and it is urgent for students to learn more about the functions of discourse markers and increase their awareness of discourse markers so as to improve their reading comprehension. In spite of these findings, this study is far from perfect due to its limited data. Therefore, in future study, more participants should be involved, and more tests with more questions related to DMs should be conducted. In a word, there is much to be done in the future before we can make this study perfect.

\section{REFERENCES}

[1] B. Fraser, "What are discourse markers?” J. of Pragmatics. Amsterdam, vol. 31, pp. 931-952, July 1999.

[2] D. Schifrin, "Conversational coherence: the role of well," Language. Washington, vol. 61, pp. 640-667. September 1985.
[3] D. Blakemore, "So as a constraint on relevance," in Mental Representations: The Interface between Language and Reality, Ruth M. Kempson, Ed. Cambridge University: Cambridge Press, 1988, pp. 183-195.

[4] D. Schiffrin, Discourse Markers. Cambridge: Cambridge University Press, 1987, pp. 73-326.

[5] B. Fraser, "Types of English discourse markers," Acta Lingtuistica Hungarica. Budapest, vol. 38, pp. 19-33, January 1988.

[6] B. Fraser, 1990. “An approach to discourse markers,” J. of Pragmatics. Amsterdam, vol. 14, pp. 383-398, June 1990.

[7] Y. Ran, "A review on pragmatics of discourse markers," Foreign Languages Research. Nanjing, vol. 4, pp. 8-14, August 2000. (In Chinese)

[8] Z. He and Y. Ran, "The pragmatic constraints of discourse connectives, ” Foreign Language Teaching and Research. Beijing, vol.3, pp.1-8, June 1999. (In Chinese)

[9] X. Chen, "Using discourse connectives in composition: An investigation of Chinese learners' argumentative writing," Foreign Language Teaching and Research. Beijing, vol. 34, pp. 350-354, September 2002. (In Chinese)

[10] D. Han and Q. Lin, “A study on EFL writing of Chinese middle school students from the perspective of adaptation theory,” J. of Inner Mongolia Normal University (Educational Science). Huhhot, vol. 30, pp. 157-161, July 2017. (In Chinese)

[11] D. Han and S. Zhuang, "A study on discourse markers in EFL writing of Chinese senior middle school students,” J. of Inner Mongolia Normal University (Educational Science). Huhhot, vol.32, pp. 93-98. June 2019. (In Chinese)

[12] J. Siegel, Exploring Listening Strategy Instruction through Action Research, UK: Palgrave Macmillan, 2015, pp. 75-180.

[13] J. Zhang, "The effect of discourse markers on EFL listening comprehension of art and sciences students," Foreign Languages Education. Xian, vol. 31, pp. 61-64, March 2010. (In Chinese)

[14] L. Degand, N. Lefèvre and Y. Bestgen, "The impact of connectives and anaphoric expressions on expository discourse comprehension," Document Design. Netherlands, vol. 1, pp. 39-51. January 1999.

[15] L. Degand and T. Sanders, "The impact of relational markers on expository text comprehension in L1 and L2,” Reading and Writing: An Interdisciplinary J. Abingdon, vol. 15, pp. 739-757, December 2002.

[16] J. H. Spyridakis and T. C. Standal, "Signals in expository prose: Effects on reading comprehension,” Reading Research Quarterly. Hoboken, vol. 22, pp. 285-298, September 1987.

[17] K. K. Millis, A. C. Graesser and K. Haberland, "The impact of connectives on the memory for expository texts," Applied Cognitive Psychology. Hoboken, vol. 7, pp. 317-339, August 1993.

[18] D. Sperber and D. Wilson, Relevance: Communication and Cognition. Beijing: Foreign Language Teaching and Research Press \& Blackwell Publisher Ltd, 2 nd ed., 2001, pp. 86-109.

[19] D. Blakemore. Semantic Constraints on Relevance. Charlotte: Baker \& Taylor Books, 1987, pp. 70-77.

[20] J. Li and Y. Huang, "English L2 reading strategies in China: Review and prospect,” Foreign Languages Education. Xian, vol. 38, pp. 62-67, May 2017. (In Chinese) 